\title{
Re: Should every medical student have exposure to robotic surgery?
}

\author{
Igor Sorokin ${ }^{1}$. Noah E. Canvasser ${ }^{2}$. Jeffrey Gahan ${ }^{1}$
}

Published online: 28 November 2017

(c) Springer-Verlag London Ltd., part of Springer Nature 2017

Keywords Robotic surgery $\cdot$ Medical student $\cdot$ Exposure

In November, the Journal of Robotic Surgery (JORS) @ JRobotSurg hosted its inaugural journal club via Twitter ${ }^{\mathrm{TM}}$. This interactive platform allowed our international following to comment, question, and/or debate the featured article. In light of the upcoming residency interviews, our quarterly journal club article focused on whether medical students should be exposed to robotic surgery. A lively discussion ensued! [see supplementary Table 1].

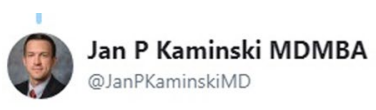

Replying to @noahcanvasser @JRobotSurg and 3 others

\section{Following}

Rob sx is like prof sport. Sure I can watch it on video, but the atmosphere and feel of OR allows $\uparrow$ engagement, ult satisfaction. \#robojc

5:46 AM - 4 Nov 2017

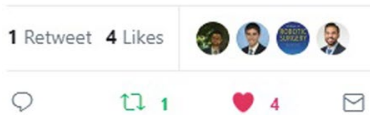

Electronic supplementary material The online version of this article (https://doi.org/10.1007/s11701-017-0764-3) contains supplementary material, which is available to authorized users.

Igor Sorokin

igsorokin@gmail.com

1 Department of Urology, UT Southwestern Medical Center, 5323 Harry Hines Boulevard, Dallas, TX 75390, USA

2 Department of Urology, UC Davis Medical Center, Sacramento, CA, USA

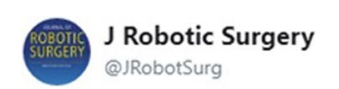

Should every medical student have exposure to robotic surgery? \#robojc discussion continues today!

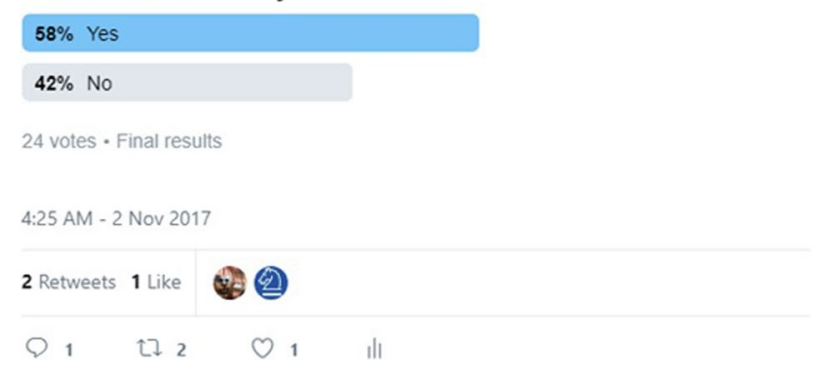

The article [1], published online in JORS in 2016, was a brief communication piece bringing to light this important topic in medical school education. The authors point out that for students going into surgical fields, "surgical bootcamps" are common in 4th year medical student rotations and incorporating robotic exposure here would be easy. For students not going into surgery, a rudimentary understanding of robotic surgery is important as every future resident or physician will care for patients who have undergone a robotic procedure. The authors concluded that initiating robotic exposure at a student level is the perfect opportunity to set the groundwork for future clinicians to provide better patient care.

The discussion was started by JORS posing the question: should every medical student be exposed to robot surgery? (1) The participants shared divergent opinions on the matter. Dr. Jonathan Ellison (@jon_ellison) thought medical students should spend less time with robots, more time with humans, and that there was no need for a formal curriculum $(2,3)$. Others shared Ellison's views noting that laparoscopic and robotic surgery are remarkably similar 
in a many ways and a curriculum differentiating the two was warranted only for those pursuing surgical specialties (4). They further opined that if internists are dealing with patients curious about robotic surgery, a webinar is a good opportunity to educate themselves (5). On the other hand, comments endorsing all medical students to be exposed to robotics were aplenty. Benefits discussed included increasing ability to answer patient's questions, to understand how technology is changing surgery, and to help select future disciplines (6-8).

The overall sentiment was that students going into surgical fields should be exposed $(9,10)$. When asked if exposure should consist of video, live surgery, or simulation, most thought exposure in all three was desirable (11-14). They argued the operating room atmosphere and vision inside the robot cannot be reproduced on video $(15,16)$. Still, others felt medical students going into surgery can pick up robotics later and that understanding the open approach in case of a complication was more important (17).

The JORS poll revealed the majority were in favor of medical students having exposure to robotic surgery (58 vs. $42 \%$ ). Of course, responses in the JORS journal club should be taken with healthy skepticism, as most followers likely have an interest in robotic surgery. Requiring formal exposure assumes that all medical students view robotic surgery as a great unknown. However, we argue that in most academic medical centers in the United States, it is hard to imagine that medical students will not have some inkling that robotic surgery exists. The potential to expose every medical student to robotic surgery is certainly feasible given the widespread dissemination of robotics in multiple surgical specialties (18). And those with a keen interest will likely have opportunities to pursue it even further. Regardless of which side you stand, the debate did bring about some interesting arguments that medical educators should consider.

\section{Compliance with ethical standards}

Conflict of interest Igor Sorokin, Noah Canvasser, and Jeffrey Gahan declare that they have no conflict of interest.

Ethical approval This article does not contain any studies with human participants or animals performed by any of the authors.

\section{Reference}

1. Hall ME, Reddy RM (2017) Should every medical student receive exposure to robotic surgery? J Robot Surg 11:375-376 